REPRESENTACIONES FINTTAS DE ANILLOS DE GRUPO DE GRUPOS A BELIA NOS.

P. Mena 1

\title{
Introduccion
}

El estudio del radical de Jacobson de un anillo de grupo ha ocupado la atencion de muchos algebristas duran-e los áltimos veinte años. El radical de Jacobson de un ani 110 da idea de "la cantidad" de representaciones irreducibles que posee el anillo. Passman [3] estudio cuándo un anilio de grupo $Q[G]$, donde $G$ es un grupo finitamente genera do, tiene muchas representaciones irreducibles finitas. Demostro que el radicaI finito de Jacobson de $Q[G]$ es cero si y s6lo si G es un grupo residualmente finito.

El proposito de este articulo es dar algunos resultados elementales sobre las representaciones finitas de un ani $110 \mathrm{~K}: \mathrm{G}$. . Una ampliacion de este trabajo es una parte del capitulo I de [2].

\section{Radical Ejpito.}

Sean $K$ un cuerpo y $G$ un grupo. K [Gj denota el anillo del grupo $G$ sobre el cuerpo $\mathrm{k}$. Todos los cuerpos que consideramos son de caracteristica cero.

Una representación

$$
P: K[G] \rightarrow E n_{K}(V)
$$

se dice que es finita si $v$ tiene dimension finita como K-es pacio vectorial.

Denotaremos por $\mathrm{R}(\mathrm{K}[\mathrm{G}])$ Ia intersección de los núcleos de todas las representaciones finitas de $K[\mathrm{G}]$. 
El radical finito $f^{i *}-R\left(\mathrm{~K}_{1} \mathrm{G}_{j}^{*}\right)$ es, siguiendo la notación de $\{3\}$, la intersección de los núcleos de todas las representaciones irreducibles $Y$ finitas de $K[G]$.

con la notacion anterior tenemos que

$$
R(K[G]) \subseteq f^{*}-R(K[G j) \quad J(K \mid G]) \subseteq f^{* H}-R(K F G j)
$$

donde $J(K[G i ̣)$ es el radical de Jacobson de $K[G]$. En el caso de que todas las representaciones irreducibles son finitas tenemos que

$$
J(K[G])=E^{*}-R(K[G])
$$

Si $G$ es un grupo, $\underline{R(G)}$ denotark la interseccion de to dos los subgrupos normales de indice finito en $G$. Si $G$ es un grupo abeliano es facil demostrar que $R(G)=n \geq 1 G^{n}$, donde $\mathrm{G}^{\mathrm{n}}=\left\langle\mathrm{x}^{\mathrm{n}}, \mathrm{x} \in \mathrm{G}\right\rangle$. Un grupo $\mathrm{G}$ se öice que es residualmente finito si $R(G)=\langle 1\rangle . G^{+}$denotará el conjunto de Ios elementos de $G$ de orden finito.

EI ideal aumentador, $w(K[G])$, de $K \mid G]$ es el nucleo de la pro yeccion $K[G]^{\prime} \rightarrow K$ en la que $\sum_{x \in G} a_{x} \cdot x \mapsto \sum_{x \in G} a_{x} \cdot$ En general si $H \varangle G$ la proyección natural $G \rightarrow G / H$ extiênde a un epimorfismo $\mathrm{K}[\mathrm{G}]^{*} \rightarrow \mathrm{K}[\mathrm{G} / \mathrm{H}]$ cuyo núcleo es el ideal aumen tador de $\mathrm{K}[\mathrm{H}]$ en $\mathrm{K}[\mathrm{G}] \mathrm{y}$ se denota por w $(\mathrm{K}[\mathrm{H}]) \mathrm{K}[\mathrm{G}]$.

1. I Lema (Wallace [5]). Sean G un grupo residualmente finito $y$ $A^{n}$ la coleccion de sus subgrupos nomales de sndice finito. Entonces $\cap(j)(K[N]) \quad K[G]=(0)$. $N \in \mathcal{N}$ como consecuencia de este lema obtenemos: 
1.2 Lema. Dado K/G; se cumple que:

$$
\left.R\left(K_{:}^{\prime \prime} G_{j}\right) \subseteq f^{* \prime}-R(K: G]\right) \subseteq u(K[R(G)]) K(G j \cdot
$$

Demostracion. Supongamos primero que $G$ es residualmente finito. Para cada subgrupo normal de indice finito $N$ sea $\left.K_{i}^{r G}\right] \rightarrow K[G / N]$ la proyeccion natural. Del Teorema de Maschke resulta que $E^{*}-R(K[G / N])=(0 ; y$ por tanto $f^{*}-R(K[G]) \subseteq \cap \omega(K[N]) K[G]$. DeI Lema 1.1 se sigue que $E^{*}-R(K[G])=(0)$.

Sea ahora $G$ un grupo arbitrario. El grupo $G / R(G)$ es resi dualmente finito, luego $f^{*}-R(K[G / R(G)])=(0)$. De ahi que $f^{*}-R(K[G]) \subset$ w $(K[R(G) j) K[G]$. El resultado se sigue, ya que trivialmente $R\left(K[G] \subseteq E^{*}-R(K[G))\right.$.

Un grupo $G$ se llama $K-l i n e a l$ si $G \subseteq G L(n, K)$ para cierto entero $n$.

Consideremos las siguientes clases de grupos

$$
\begin{aligned}
& G_{K}: \text { la clase de los grupos K-lineales } \\
& R_{E} \text { : la clase de los grupos residualmente Einitos. }
\end{aligned}
$$

Entonces tenemos:

1.3 Lema. Supongamos que $\zeta$ es una clase de grupos cerrada por cocientes $y$ tal que $\xi \cap G_{K} \subset R_{F}$. Entonces para cada $G \varepsilon \zeta$ se tiene que $R(K[G])=f(K[G])=w(K \cdot R(G) I) K[G]$.

Demostracion: Teniendo en cuenta el Lema 1.2 basta probar que $w(K[R(G)]) K[G j \subseteq R(K[G])$. Sea $g \in R(G) Y \rho: K[G] \rightarrow M(n, K)$ una representacion finita de $K[G]$. El grupo $\rho(G) \notin \zeta \cap G_{K} y$ en 
consecuencia es residualmente Finito, asf $\rho(g)=1$. Puesto que eI conjunto $\{1-g \mid g \in R(G)\}$ genera el ideal w(K[R(G) $]) K[G]$ se tiene que $u(K[R(G)]) K[G] \subseteq R(K[G])$.

Ejemplos de grupos que satisfacen las hipotesis del Lema 1.3 son:

(1) los grupos finitamente generados (Malcev Th.4.2[4]).

(2) los grupos de torsion (si $K=$ Q) (Th. 9.33, [4]).

Un grupo $G$ se dice que es un F.C. grupo si cada elemento tiene s6lo un número finito de conjugados.

1.4 Proposicion. Sea $K$ un cuerpo tal que los grupos multiplica tivos de las extensiones finitas de $K$ son residualmente $f i-$ nitos. Sea G un F.C. grupo. Entonces $E^{*}-R(K[G])=[(K[R(G)]) K[G]$. Demostración. Sea $P: K[G] \rightarrow A$ una representacion irreducible $y$ finita de $K[G]$. $\rho(G)$ es un F.C. grupo K-lineal, por tanto es una extension finita de su centro $z(p(G))($ cor. 5.6.[4]).

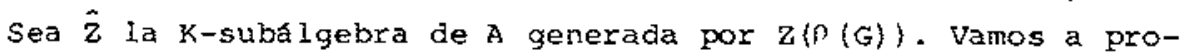
bar que $\hat{z}$ es semisimple. En efecto, por ser $\hat{z}$ central en $\rho(K[G])$ podemos escribir que $J(\hat{Z}) \rho(K[G])=\rho(K[G]) J(\hat{Z})$ y pues to que $J(\hat{Z})$ es nilpotente resulta que $J(\hat{Z}) \rho(K[G])$ es un ideaI nilpotente de $\rho(K[G])$. Luego $J(\hat{Z}) \rho(K[G])=(0)$, ya que $\rho(K[G])$ es simple. Entonces $\hat{z}$ es isomorfo con un producto de cuerpos $L_{1} \times \ldots x I_{n}$, sada $L_{1}$ es una extension finita de $K$. Por tanto $\mathrm{z}(0(\mathrm{G})) \hookrightarrow \mathrm{L}_{\mathrm{j}}^{*} \times \ldots \times \mathrm{L}_{\mathrm{n}}^{*}$ y de I'as hipotesis se deduce que $Z(0(G))$ es residualmente Einito. Como $[0(G): Z(n(G))]<\infty$ resulta que $n(G)$ es residualmente finito. Entonces $\omega(K[R(G)]) K[G] \subseteq \operatorname{Ker} \rho . y$ como $\rho$ era una representacion finita arbitrarla se tiene que

$$
\omega(K[R(G)]) K[G] \subseteq R(K[G])
$$


Hay ejemplos de cuerpos cuyos grupos multiplicativos son re sidualmentefinitos y sin embargo los grupos multiplicativos de algunas de sus extensiones finitas no son residualmente finitos. Basta considerar el cuerpo

$$
K=Q\left(\cos \pi / 2^{n}, \text { para todo entero } n \geq 1\right)
$$

y la extension $\mathrm{L}=\mathrm{K}(\mathrm{i})$.

$S i \mathrm{~K}$ es un cuerpo de numerog el grupo. multiplicativo $\mathrm{K}^{*}$ es suma directa de grupos ciclicos (Th. 127.2, [1]) y en particular residualmente finito.

1.5 Corolario. Sea G un F.C. grupo. Entonces

$$
f^{*}-R(Q[G])=w(Q[R(G)]) Q[G]
$$

El corolario 1.5 es cierto para otras clases de grupos, por ejemplo para grupos localmente resolubles.

En general, para grupos arbitrarios, el corolario 1.5 no es cierto. Por ejemplo si $n>1$ y $G=G L(n, 0)$, la identidad $\mathrm{GL}(n, Q) \rightarrow \mathrm{GL}(n, Q)$ induce una representación irceducible $Q[G]-M(n, Q)$. Si el corolario 1.5 fuese cierto para $G$ tendriamos que $R(G)=\langle 1\rangle$ Io cual es absurdo.

2. $R(Q[G])$. Supondremos que $G$ es un grupo abeliano y demostra remos el siguiente

2. I Teorema. $\left.R(Q[G])=(Q)\left[R(G)^{+}\right]\right) Q[G]$.

Demostracion. Procederemos en varias etapas.

(1) $\mathrm{G}=\mathrm{Q}^{+}$(eI grupo aditivo de Q). En este caso hemos de probar que $\mathrm{R}(\mathrm{Q}[\mathrm{G}])=(0)$. 
consideremos las aplicaciones $\left\{\rho_{n}\right\}_{n \in \mathbb{N}}$ definidas por

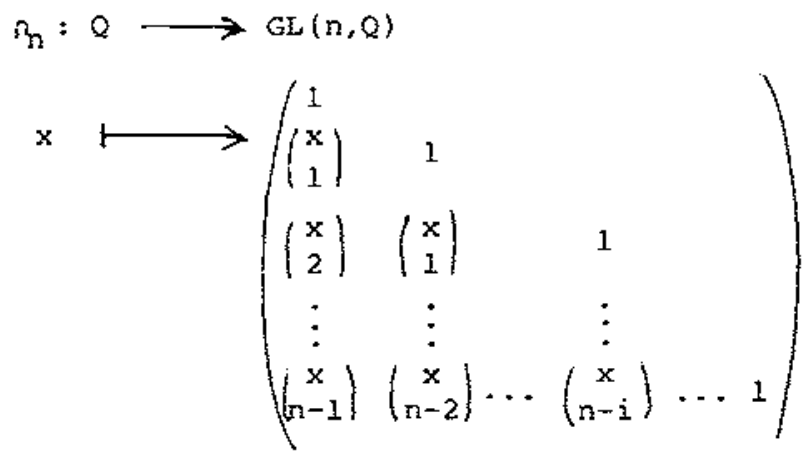

es facil comprobar que cada $o_{n}$ es un homomorfismo de grupos. Si $x \neq 0$ el polinomio minimo de la matriz $n_{n}(x)$ es $(x-1)^{n}$. Sea $\alpha \in \cap \operatorname{ker} \rho_{n}^{*}$ (donde $\rho_{n}^{*}: 0[0], M(n, Q)$ es la extensior. $n \geq 1$ de $\rho_{n}$ a $\left.Q(Q]\right), \alpha=\sum_{i=1}^{n} q_{i} \cdot x_{i} \in Q[Q]$. Puesto que $Q$ es 10 calmente ciclico sea $x \in Q$ tal que $\langle x\rangle=\left\langle x_{1} \ldots, x_{n}\right\rangle$. Si $x=0$ se tiene que $a=\left(\sum_{i=1}^{n} q_{i}\right)$. Ya que $1 \notin \bigcap_{n \geq 1}$ Ker $q_{n}^{*-}$ es claro que $\sum_{i=1}^{n} q_{i}=0$ y $\alpha=0$. Supongamos pues que $x \neq 0$ y escri bamos $a=\sum_{i=1}^{n} q_{i} \cdot \lambda_{i} \times$ para enteros $\lambda_{i}$ convenientes. Conside remos la fraccion racional $q(x)=\sum_{i=1}^{n} q_{i} x^{\lambda}$. Multiplicando $q(x)$ por su conveniente $x^{h}$ se obtiene un polinomio $p(x)=x^{\breve{h}} q(x)$. Sea $m_{0}$ el grado de $p(x)$. Sea $m>m_{0}$, ya que a $\in \cap$ ker $\rho_{n}^{*}$, tene$\mathrm{n \geq 1}$

mos que $\sum q_{1} \rho_{0}(x)^{\lambda_{1}}=0$, o sea $p\left(\rho_{0}(x)\right)=0$. ya que el poino 
mio minimo de $\eta_{0}(x)$ es $(x-1)^{m}$ y $m>m_{0}$, se sigue que $p(x)=0$. Luego $q_{i}=0,1 \leq i \leq m, y a=0$.

(2) $G=\sum_{I} Q$. Sea $\alpha \in Q\left[\sum_{I} Q\right], \alpha=\sum_{i=1}^{n} q_{i} \cdot x_{i} \cdot$ Sea $J_{\subseteq} I$ un conjunto finito tal que $\alpha \in Q\left[\sum_{J} Q\right]$. Sea $\pi: \sum_{I} Q \rightarrow \sum_{J} Q$ la proyeccion natural. Tenemos que $\pi\left(x_{1}\right)$, I $\leq i \leq n$, tiene una expresion del tipo $\pi\left(x_{1}\right)=\left(x_{1}, \ldots, x_{1},\right)$ donde $m=|J|$. Sean $e_{1},=\pi\left(x_{1}\right)-\pi\left(x_{j}\right) \quad 1 \leq i \neq j \leq n$. Ya que $e_{1 j} \neq 0$ existe un hiper plano de $Q^{\mathrm{m}}$ que no contiene ningún $e_{1 j}$. En otras palabras existen $\lambda_{1}, \ldots, \lambda_{v} \in Q$ tales que $\bar{x}_{1}=\sum_{j=1}^{m} \lambda_{f} x_{t j}, 1 \leq i \leq n$, son todos distintos. Definimos $w: \sum_{\mathcal{J}} \mathrm{Q} \rightarrow Q$ por $w\left(\mathrm{y}_{1}, \ldots, \mathrm{y}_{0}\right)=\sum_{i=1}^{\mathrm{m}} \lambda_{1} y_{1}$. Supongamos ahora que a $\in R\left(Q\left[\sum \quad 0\right]\right)$. Entonces si $\omega^{*}$ y $\pi^{*}$ son las respectivas extensiones $I$ de $\omega y \pi$ al anillo de grupo. se tiene que $\left(\omega^{*} \pi^{* *}\right)(\alpha) \in R(Q[Q]) y$, de $(1)$, tenemos que $\left(\omega^{* *} \pi^{*}\right)(\alpha)=0$, o sea $0=\left(\omega^{* 6} \pi^{*}\right)(\alpha)=q_{1} \cdot \bar{x}_{1}+\cdots+q_{n} x_{n}$. Por construccion los elementos $\bar{x}_{1} \ldots, \bar{x}_{n}$ son distintos, enton$\operatorname{ces} q_{1}=\ldots=q_{a}=0 \quad y \quad a=0$.

(*) Observemos que si $\mathrm{R}(\mathrm{K}[\mathrm{H}])=0$ y $[\mathrm{G}: \mathrm{H}]=\mathrm{n}<\infty$. entónces $R(K[G])=0$. En efecto $K[G]$ es un $K[H]-m b d u l o$ Iibre (por la izquierda) de dimensión $n$. Entonces $k[G] \hookrightarrow M(n, K[H])$.

(3) G es libre de torsion. Entonces $G C \sum_{I} Q$ para un conjunto de Indices I conveniente. como $R(Q[G]) \leq R\left(Q\left[\sum Q\right]\right)$ el resultado se sigue de $(2)$.

(4) $G^{+}$es residualmente finito. Sea $\alpha=\sum q_{s} \cdot x_{1} \in R(Q[G])$. 
Ya que $\mathrm{G}^{+}$es residualmente Einito existe su subgrupo $\mathrm{N}$ de $\mathrm{G}^{+}$ tal que $\left[G^{+}: N\right]<\infty$ y $x_{1} x_{j}^{-1} \neq N$ para $i \neq j$.

Supongamos primero que $\mathrm{G}^{+}$es un sumando directo de $\}$, es decir $\mathrm{G}=\mathrm{G}^{+} \times \mathrm{T}$. Entonces $\mathrm{G} / \mathrm{N} \cong \mathrm{T} \times \mathrm{G}^{+} / \mathrm{N}$. Por tanto $\mathrm{G} / \mathrm{N}$ es una extension finita del grupo ' $\mathrm{I}$ que es libre de torsion. De $(*) \quad Y(3)$ se deduce que $R\left(Q_{(}(j / N)\right)=(0)$. En la proyeccion natural $Q[G] \rightarrow$ of $G / N]$ se tiene que $a \rightarrow \bar{a}:=\Sigma_{1} q_{f} \cdot \bar{x}_{1} \quad y$ los elementos $\bar{x}_{1}$ son distintos. Como $\left.\bar{a} \in R(Q ; G / N]\right)=(0)$ tenemos $\mathrm{q}_{\mathrm{f}}=0 \mathrm{y} \mathrm{a}=0$. Supongamos ahora que $\mathrm{G}^{+}$no es necesariamente un sumando directo de G. Tenemos que el subgrupo de torsion de $\bar{G}=G / N$ es finito. Sea $n \geq 1$ un entero tal que $G^{n}$ es $I i-$ bre de torsion. Entonces $G \leftrightarrow \bar{G}^{n} \times \bar{G}^{-n}$. El resultado se sigue de la primera parte.

(5) $G$ es un grupo abeliano arbitrario. Se deduce de (4) que $\left.R\left(Q / G / R(G)^{+}\right]\right)=(0)$. De ahi que $R(Q[G]) \leq 4\left(Q\left[R(G)^{+}\right\}\right) Q[G]$ : La otra inciusion se sigue del hecho que los grupos Q-lineales de torsion son finitos (TH. 9.33, [4]).

B I B $\quad$ L $I$ I O

[1] L. Fuchs, Infinite Abelian Groups. Acad. Press, 1970.

[2] P. Mena 1, Tesis, presentada en la Universidad Autonoma de Barcelona, 1977 .

[3] D.S. Passman, On groups with enough finite representations. Proc. Amer. Math. Soc., 14 (1963), 782-787,

[4] B.A.F. Wehrfritz, Infinite Linear Groups, Springer-verlag, 1973.

[5] D.A.R. Wallace, The Jacobson Radinals of the Group Algebras of a Group and of Certain Normal subgroups. Math. Zeitschr, 100 (1967), 282-294. 